\title{
Narratives for drug design
}

James Bown

Alexey Goltsov

This is the Author's Accepted Manuscript of the book chapter:

Bown J., Goltsov A. (2018) Narratives for Drug Design. In: Walsh R., Stepney S. (eds) Narrating Complexity. Springer, Cham

The final publication is available at Springer via https://doi.org/10.1007/978-3-319-64714-2 18 


\section{Narrating drug design}

James Bown

School of Arts, Media and Computer Games

School of Science, Engineering and Technology

Abertay University, UK

Alexey Goltsov

School of Science, Engineering and Technology

Abertay University, UK

\section{Narratives and cancer patients}

The management of patients with long-term conditions is one of the major challenges facing healthcare systems worldwide (United Nations General Assembly 2013). A long-term condition is a condition for which there is no cure; rather, longterm conditions must be managed through a range of treatment options including drugs (King's Fund 2012). Long-term conditions include diabetes, hypertension, chronic kidney disease and cancer, and management accounts for a substantial proportion of health service resources. For example, in the UK long-term condition patients account for $30 \%$ of the patient population and $70 \%$ of the healthcare spend (Department of Health 2012).

Patients living with long-term conditions play an important role in management of their condition, and Coulter et al. (2015) provide a comprehensive review of the role of personal care planning in the management of long-term conditions. They describe personal care planning as a "collaborative process used in chronic condition management in which patients and clinicians identify and discuss problems caused by or related to the patient's condition, and develop a plan for tackling these" (Coulter et al. 2015). For personal care planning to be effective, patients must be supported in the difficult decisions they make in respect of lifestyle choices and treatment options.

Cancer is one of the fastest rising long-term conditions (Department of Health 2012). Cancer is one of the leading causes of death worldwide, with approximately 8 million deaths recorded in 2012 and a 70\% rise in new cases expected over the next 20 years (Stewart \& Wild 2014). Narrative has been shown to have value in the prevention of cancer through effective communication of risk. Janssen et al. (2013) provide a useful review of narrative in healthcare, noting that "by providing vivid information about the antecedents and the consequences of a health problem, narrative health information improves the extent to which people are able to imagine themselves developing a certain disease, which in turn may 
influence their risk judgments" (Janssen et al. 2013). Their own study explored the effects of risk communication to regular sunbed users in narrative and nonnarrative forms. Results showed that, compared with non-narrative information forms, narrative information promoted an increased feeling of skin cancer risk with respect to sunbed use in participants, and participants could more readily image themselves developing skin cancer.

Narration can also support patients when making decisions on treatment options. Shaffer et al. (2013) report on the effect of process-focused and experiencefocused narratives on the patient decision-making process. Process narratives are designed to "prime participants to follow a particular decision process ...[and] would most commonly entail patients considering additional dimensions of the decision process that they might not have considered otherwise" Shaffer et al. (2013). Experience narratives are designed to "increase knowledge and the perceived ability to imagine future health states ... [which] could result in increased decisional satisfaction and an improved ability to make affective forecasts (i.e. forecasts of future feelings)" Shaffer et al. (2013). Importantly, neither process or experience narratives are thought to bias healthcare decisions, but to promote consideration of a broader set of issues than they might have otherwise or improve understanding of treatment outcome respectively. Focusing on breast cancer treatment decisions, and through a carefully designed test with control conditions, results of the study revealed that participants exposed to processfocused narratives spent more time searching for information relating to key aspects of treatment that were discussed in the narratives. Participants exposed to experience-focused narratives were more confident in and satisfied with their treatment decisions.

We propose that narrative can also inform clinicians' understanding of cancer. We base this proposition on the following observations: 1) cancer is a complex system; 2) effective drug design depends on understanding that complex system; and 3) narratives can inform our understanding of complex systems. The remainder of this chapter explores this proposition by unpacking these three observations in turn.

Section 2 considers cancer as a complex system. We unpack some of the complexities associated with cancer as a system of interacting cells in the context of normal tissue. We pay particular attention to one level of functioning in cancer cells - that of the intracellular signalling network that represents the biochemical interactions among different species in the cell. It is these biochemical interactions that ultimately dictate cell fate.

Section 3 explores this signalling network in the context of anti-cancer drug targets. Because of their role in cell fate, some signalling network components 
provide potentially useful drug targets for anti-cancer therapy. However, these drug targets are situated in the context of a topologically complex and dynamic network, and we consider how anti-cancer drugs seek to restore normal functioning in cell signalling networks and explore how therapy design is impeded by the complexity of the cellular system.

Section 4 sets out what narrative might offer to support the process of drug design, both providing an example of work done to date that might serve as a foundation for narrating complexity and speculating on the contribution of narrative to anti-cancer treatment. In Conclusion we suggest possible ways of using this work combined with that of others to begin to consider narrating drug design.

\section{Cancer as a complex system}

Cancer is not a single disease; it is a broad class of diseases of over 200 types (Cancer Research UK 2016), characterised by functional dysregulations within and surrounding affected cells, tissues and organs (Bown et al. 2012) as outlined in subsequent sections. Cancer originates from the aberrant behaviour of a single cell or region of cells that over time gives rise to an observable anomalous tissue structure in the form of a tumour, and can progress to non-local spread through the blood stream or lymphatic system.

Cancer is therefore an emergent system where local (cell) scale processes lead to system-scale patterns in local cell populations, tissue structures, organs and ultimately in the body as a whole. As explored below, those tissue patterns in turn impact cellular processes. Moreover, there is increasing awareness of the heterogeneities in cancer: tumours comprise multiple cell types; patterning in tissue is likewise heterogeneous. That cancer is emergent at multiple scales and highly heterogeneous makes treatment very challenging.

In 2000, Hanahan and Weinburg (2000) set out six biological hallmarks of cancer that have helped frame investigations and interpretation of findings. Here, these hallmarks are only briefly outlined and indeed greatly simplified since they provide a contextual backdrop to the challenge of drug design. Hanahan and Weinburg (2000) provide a rich description of all six hallmarks for the interested reader.

Normal cells regulate the processes of growth and division and pre-programmed cell death, responding to spatially and temporally structured external signals that cue the cell to grow and information on mechanical stresses from the environment and from other cells (note there are a wide range of other factors involved). In effect, external signals are read in through receptors on the cell wall and 
processed by the cell to drive behaviour. This cellular processing enables tissue to maintain consistent and properly functioning structures.

Cancer arises from perturbations in the processing of these signals, and such perturbations can lead to cells that are not well regulated by external stimuli. This dysregulation can confer cells with the six hallmarks of cancer:

- Increased proliferation, where cells divide far more frequently than they should;

- Unsuppressed growth, where cancer cells can grow in structural forms inconsistent with normal tissue (e.g. where mechanical pressures are larger);

- Resistance to cell death, a natural and pre-programmed mechanism to promote cell turnover and maintain a healthy population of cells;

- Replicative immortality, through a combination of the above three hallmarks and through changes in the mechanisms cells use to control the number of possible divisions;

- Sustained angiogenesis, meaning that cancerous tissue can encourage development of structures able to supply oxygen and nutrients;

- Invasion and metastasis, where tumour masses can move into adjacent tissue and into distant regions by changing the physical coupling of cancer cells to their microenvironment.

Tumours thus originate from and are sustained by dysregulations within the signal processing within the cell, which confer on that cell particular ecological and evolutionary advantages. The resulting pattern at the tissue scale is the emerging tumour of cancer cells in the environmental context of normal tissue. An important observation is that cross-scale feedback occurs through competition for resource and space. Resource competition occurs because there is limited oxygen and nutrients yet there are increasingly more cells in the developing cancerous tissue structure. This growth is occurring in a limited space and the mechanical stresses on cells caused by too much growth in too little space are converted into biochemical signals and can actually promote further signal transduction and these stresses drive proliferation over time (see Jaalouk and Lammerding 2009 for a review).

Kreeger and Lauffenburger (2009) provide an excellent review on the challenges posed when trying to unravel both the origins and consequences of such dysregulation in cancer cells. A key observation is that cell behaviour is controlled by a mix of genetic alterations and environmental context, and that the "greatest amount of information concerning phenotypic behaviour resides in the realm comprehending both genomic and environmental effects: dynamic protein network operations" (Kreeger and Lauffenburger 2009). 
These protein networks, or signalling networks, provide a mechanistic connection between external signals received at the cell surface and the cell nucleus (Cooper 2000). These signalling networks represent the biochemical species that interact to form new compounds in order to process external signals. The nodes in the network represent the compounds that are formed and broken down in space over time as the cell processes extracellular signals and it is the result of this processes that drives the behaviour of the cell.

Signals propagate through these networks and cancerous behaviours, i.e. the hallmarks, are often associated with measurable differences in the proteins that make up these pathways. Accordingly, amongst the myriad of levels of organisation of cellular, tissue and environmental factors, signalling networks are a promising route for drug design. However, signalling networks attract complexities in of themselves.

\section{Signalling networks and anti-cancer drug design}

As noted above, signalling networks transduct external stimuli, including growth factors and anti-cancer drugs, for processing by the cell nucleus. Importantly, these networks do not operate in isolation; networks are interconnected and this complicates their study.

Figure 1 (from Hu et al. 2013) is illustrative of such a network, and shows two key, interconnected signalling pathways that are implicated in some of the hallmarks of cancer: pre-programmed cell death, cell proliferation and cell growth. These pathways are regulated by growth factor receptors (HER2 and HER3 in Figure 1). These two receptors regulate signalling in the PI3K/PTEN/AKT and Ras/Raf/MEK/ERK pathways that control cell survival, growth and proliferation. The details of the molecular species that comprise this network are beyond the scope of this Chapter. Important here are several topological features that give rise to complex, emergent behaviours. Note these features are a defining property of many signalling networks in biological sciences (Bown et al. 2012) - complexity arising from network topology is prevalent and not limited to this exemplar network or indeed to cancer. 


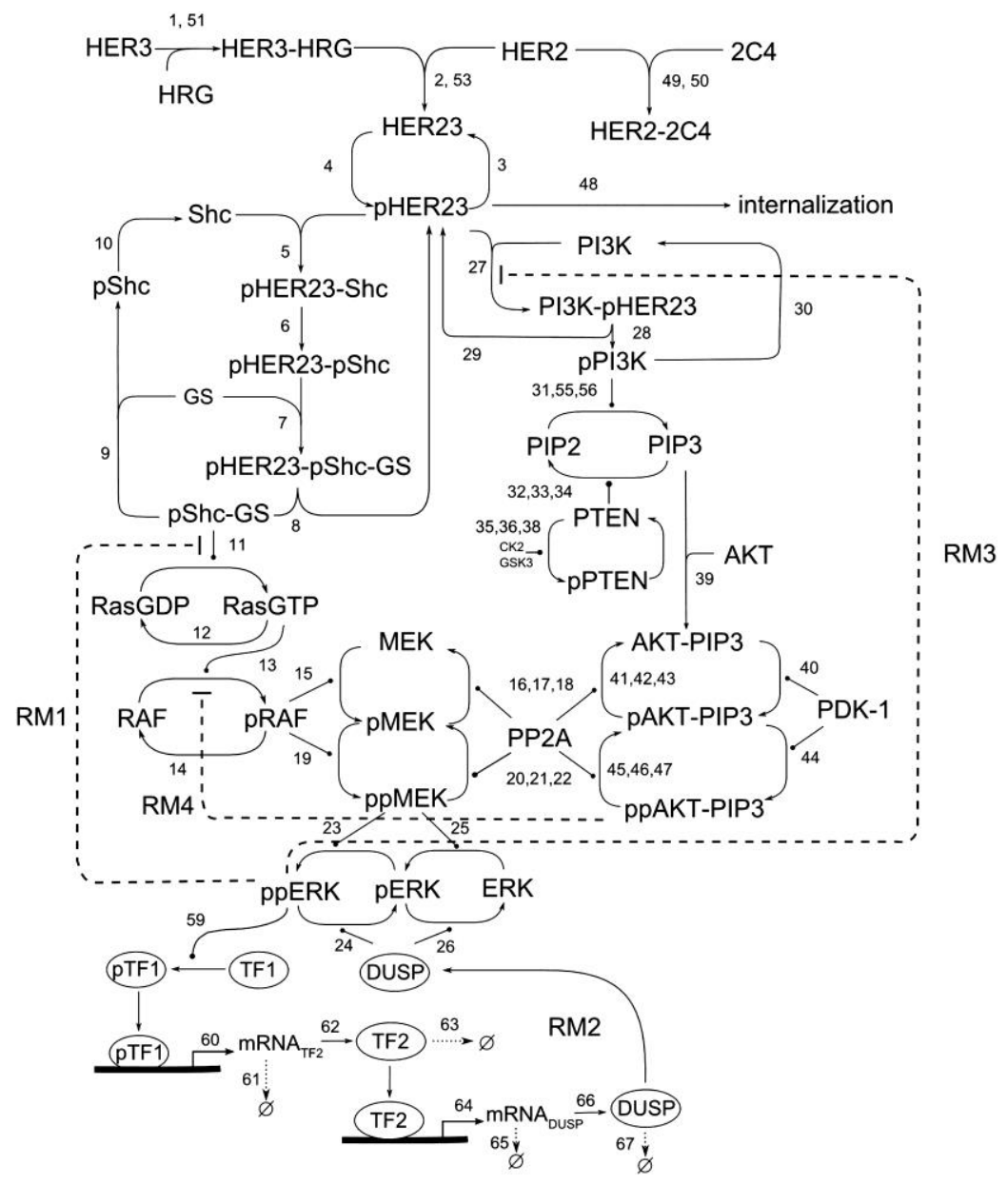

Figure 1: Cellular signalling pathways (reproduced from Hu et al. 2013). The two pathways RAF/MEK/ERK (left hand side) and PI3K/PTEN/AKT (right hand side) are interconnected by element PP2A. RMI shows a feedback loop. Additional crosstalk is shown by RM3 and RM4. Numbers refer to the underlying equation set (see discussion on interactive media below).

Figure 1 shows pathway cross-talk and feedback loops, within and between networks. Cross-talk is shown towards the centre of the network by the PP2A enzyme, a known regulator of a wide range of cellular processes. This enzyme is connected to the AKT-PIP3 complex, which drives cell survival and growth, and the MEK complex, implicated in cell proliferation and differentiation among other processes. A cell has a limited amount of PP2A at any one time and in this particular cross-talk example, an increase in signalling activity in one pathway that interacts with this limited amount of PP2A causes an inhibition of signalling activity in the other pathway. Cross-activation, rather than the cross-inhibition shown here, is also observed in other pathways.

Signalling is further complicated by the feedback loops shown in the network (Figure 1, dotted lines). Feedback loops are another pervasive feature of biological networks. Feedback loops have a regulatory role in such networks, helping to keep 
some intracellular conditions constant in the face of any perturbation. This network describes oscillations in AKT and ERK signalling pathway outputs, and these oscillations can be controlled by varying the strengths of the feedback loops in the networks.

These topological complexities on the one hand provide signalling networks with marked robustness to external stimuli, maintaining proper functioning in the face of noisy inputs, yet on the other hand confer exquisite sensitivity to key variations in those inputs. Accordingly, networks can be either sensitive or resistant to small changes in input signals: sensitivity means that small, localised changes can have a pronounced impact on non-local network functioning; resistance means that network functioning is resilient to such change.

This emergent phenomenon has implications in anti-cancer drug design (see below) but also in cancer-associated mutations. Figure 2 (from Goltsov et al. 2014) shows the impact of a cancer-associated mutation on network sensitivity in the form of a heatmap. The heatmap shows the sensitivity of 19 different entities in the signalling network, where light grey indicates high sensitivity and dark grey indicates low sensitivity, i.e. resistance. In normal functioning (Column 1 in Figure 2), the network sensitivity heatmap is mainly mid to light grey, indicating a network that is sensitive to change but not dramatically so. Mutations can be introduced into the model to represent biological mutations in the cell. One single mutation in a key network node results in a network that is largely insensitive to change (Column 3 in Figure 2 is mainly dark grey and black), i.e. a local change results in a marked non-local change in sensitivity.

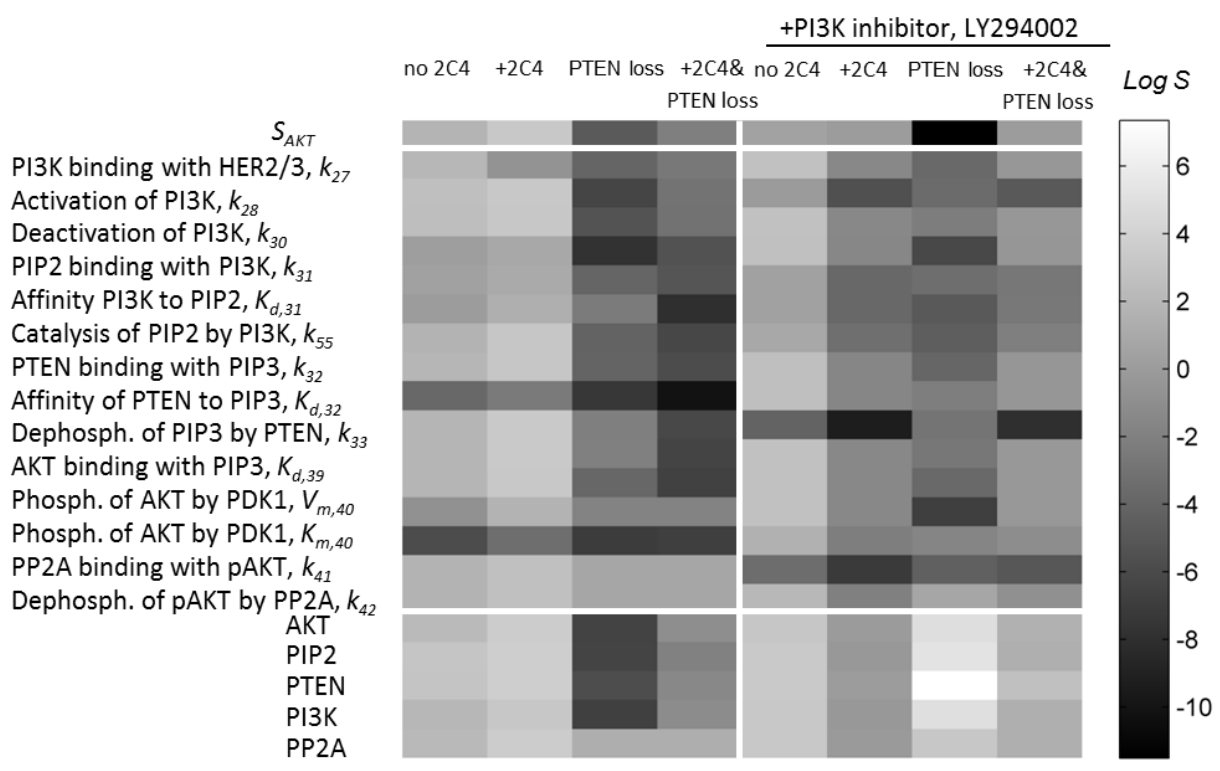


Figure 2: Heatmap of sensitivity of different entities in the signalling network (adapted from Goltsov et al. 2014). Column 1 shows the network in normal functioning, and when an anti-cancer drug is applied the network increases in overall sensitivity (Column 2). When a cancer-associated mutation is introduced the network is insensitive without or with the anti-cancer drug (Columns 3 and 4 respectively). The addition of a second drug in combination with the first (Columns 5-8) restores sensitivity to the anti-cancer drug (Column 8).

To complicate cellular signalling further still, there is an increasing awareness that the network topology itself is not fixed (Lee et al. 2012). The network topology represents the interactions among different species in the cell, and the network changes in structure over time. These changes occur because different parts of the network interconnect and disconnect as the cell responds to acquired mutations and significant changes to external stimuli such as anti-cancer drug treatments. For example a cell can become resistant to the effect of a drug through these changes.

Anti-cancer drug treatments are typically in the form of a "targeted cancer therapy", a kind of therapy that is designed to disrupt aberrant behaviour in cellular signalling networks, either in an effort to restore normal functioning or to at least suppress cancerous behaviours in cells, by targeting a particular node in the network. Drugs are typically designed in a single-target-single-drug paradigm (Medina-Franco et al. 2013), i.e. a drug is designed to target a specific site in a network. However, single therapies have had only limited success (Singer et al. 2008), with patients either failing to respond at all or developing resistance to the drug effect over time.

This is, in part, because the single therapies are acting in the context of a range of mechanisms that compensate for and adapt to perturbations (here, drug action): these mechanisms include cross-talk, feedback loops, differential sensitivities to change across the network and changes in network structure in response to drug action. This means that targeted therapies can impact beyond their point of application, and often in ways that are difficult to anticipate (Bown et al. 2016). These features then limit efficacy of any single therapy, and patient resistance to a drug is a key challenge in anti-cancer therapy design.

There is increasing evidence from both in vitro and in vivo studies that combination therapy, i.e. therapy comprising more than one drug and so target more than one site in the network, is a promising route to overcome the challenge of drug resistance. This evidence base is growing continually (Chandarlapaty 2012; Chong and Jänne

2013) but typically the way in which the drugs work together to deliver improved performance is not well understood (Goltsov et al. 2014). The rational design of 
combination therapy depends on a mechanistic understanding of those networks in terms of the individual components and the way in which those components interact, locally and non-locally. Rational design needs ways of integrating, often fragmented, data that together reflects the system as a whole, or at least some representative subset of that system, and of interpreting the results of that integration.

The complexity of that integration provides an opportunity for computational models. Models can identify signalling network states that confer drug resistance or sensitivity and shed light on how to manage the transition from one state to the other through combination therapy e.g. (Goltsov et al. 2012), and propose mechanisms of combination therapy action to explain why in a model of drugs binding to signalling network nodes two drugs that are applied individually are ineffective yet when applied in combination and at the same time are effective in overcoming drug resistance (Kholodenko 2015). Thus models can contribute to rational drug design and in doing so help us understand signalling pathway complexities.

This opportunity is, however, impeded by the computational - biological discipline divide. Biologists and clinicians readily understand simple models, but simple models cannot deliver value in the face of the complexities noted above. Models that represent sufficient complexity to help understand a signalling network can be challenging for biologists to first formulate and then interpret (Janes and Lauffenburger 2013). Janes and Lauffenburger (2013) provide a review of the value of such signalling network models for experimental cell biology. They highlight that key barriers are confusions relating to the purpose of the model, predictions from the model and the wide range of modelling approaches available. The purpose should be to - try to - explain specific phenomena observed in experimentation; predictions made are often in the context of assumptions especially relating to gaps in knowledge for parameters; the selected approach needs to take account of the purpose and the available knowledge. Indeed, our own work on CoSMoS (Stepney et al. 2011) provides a framework to address exactly these barriers.

Going beyond this computational - biological discipline divide, the complexities in signalling networks run deeper than topology. Nodes, and combinations of nodes, in the network serve as switches, integrators and inhibitors, and the specific function of any given node or sub-network can be variable, contextualised by its inputs in a non-linear manner. Thus, non-linear components operate with variable function in complex networks. It then becomes impossible to describe system behaviour in linear and simple narrative. In fact, it has been suggested that rather than a node-centric view, it is likely that the dynamic features of the network itself might form the basis of drug targets rather than the network components (Behar et al. 2013). 


\section{Towards narratives for anti-cancer drug design}

We have so far established the following:

- Cancer is a complex system driven in part by aberrant cellular function;

- Signalling networks are a useful level of detail at which to study cellular function, and are themselves complex;

- Parts of those signalling networks can be targeted by anti-cancer drug therapies to seek to restore aberrant functioning;

- The signalling network is dynamic in its reaction to drugs and mutations, and its structure can change over time;

- Computational models can support our understanding of cell responses to drug action, including the various mechanisms of drug resistance;

- Computational models that are of sufficient detail to represent mechanisms of resistance can be prohibitively complex for biologists.

Here, we describe briefly an interactive visualisation technology that could provide the foundations for narratives. The use of data storytelling to communicate and stimulate insights is a growing research area (Bach et al. 2016). Segel and Heer (2010) provide a systematic review of work seeking to combine narrative and interactive visualisations, and note that while sophisticated visualisation tools might provide powerful vehicles for discovering stories narrative communication depends on more than visualisation.

Boy et al. (2015) distinguish between two types of information visualisations: explanatory and exploratory. Explanatory information visualisations are common in journalistic contexts, are typically used to support the narrative presented in the text, and have limited interactivity. Segel and Heer (2010) categorise such explanatory information visualisations as author-driven. In contrast, exploratory information visualisations require a reader-driven approach with free interactivity (Boy et al. 2015), and are motivated by provoking discoveries in the patterns of data.

We propose that our technology is a vehicle to support reader-driven narratives, but is not in of itself a narrative. This technology, SiViT (Bown et al. 2016), turns a complex model into an interactive animation, allowing the cancer specialist intuitive access to complex systems models otherwise inaccessible. SiViT is able to represent graphically the network structure of models of cell signalling, such as that described in Figure 1. The models encapsulate a system of differential equations and SiViT computes these equations and animates a simulation of the model the system dynamics. Thus SiViT provides a 'movie' of the simulation, 
showing the whole system behaviour. Moreover, each node in the network can be queried and a pop-up graph of node activity over time presented.

This is a useful contribution in respect of validation: all models depend on a set of assumptions and these assumptions can be difficult to elicit, especially in the case of complex systems models. Modelling frameworks such as CoSMoS (Bown et al. 2012) have found ways of explicating and then challenging the assumption set underpinning a complex systems model and its simulation. SiViT provides a complementary explication: simulation dynamics are animated in the hope that major departures in the model (or indeed simulation) formulation are identified.

Crucially, SiViT also allows the user to add in and then visualise the effects of cancer-causing mutations and anti-cancer drugs. Mutations associated with drug resistance can be introduced by changing simulation parameters through another drop down menu. Drugs can be added in through a drop-down menu, at a prescribed dosage at a particular time. Combinations of drugs can be added to explore the effects of different doses and of dose sequencing. These combinations can be drawn from a known set of drugs. Alternatively, new drugs can be designed by changing simulation parameters directly to simulate the effect of that designed drug.

Any simulation configuration, in terms of drugs and mutations can be compared with another (one) simulation configuration. In pairwise comparisons, the two configurations are defined as Control and Experiment and the visualisation is a mix of red, blue and white. The colour of each node and edge component is set by whether the value of the Control component is greater than, less than or equal to the value of the corresponding component in the Experiment, with colour intensity proportional to this difference. Figure 3 shows a signaling network visualisation using SiViT. 


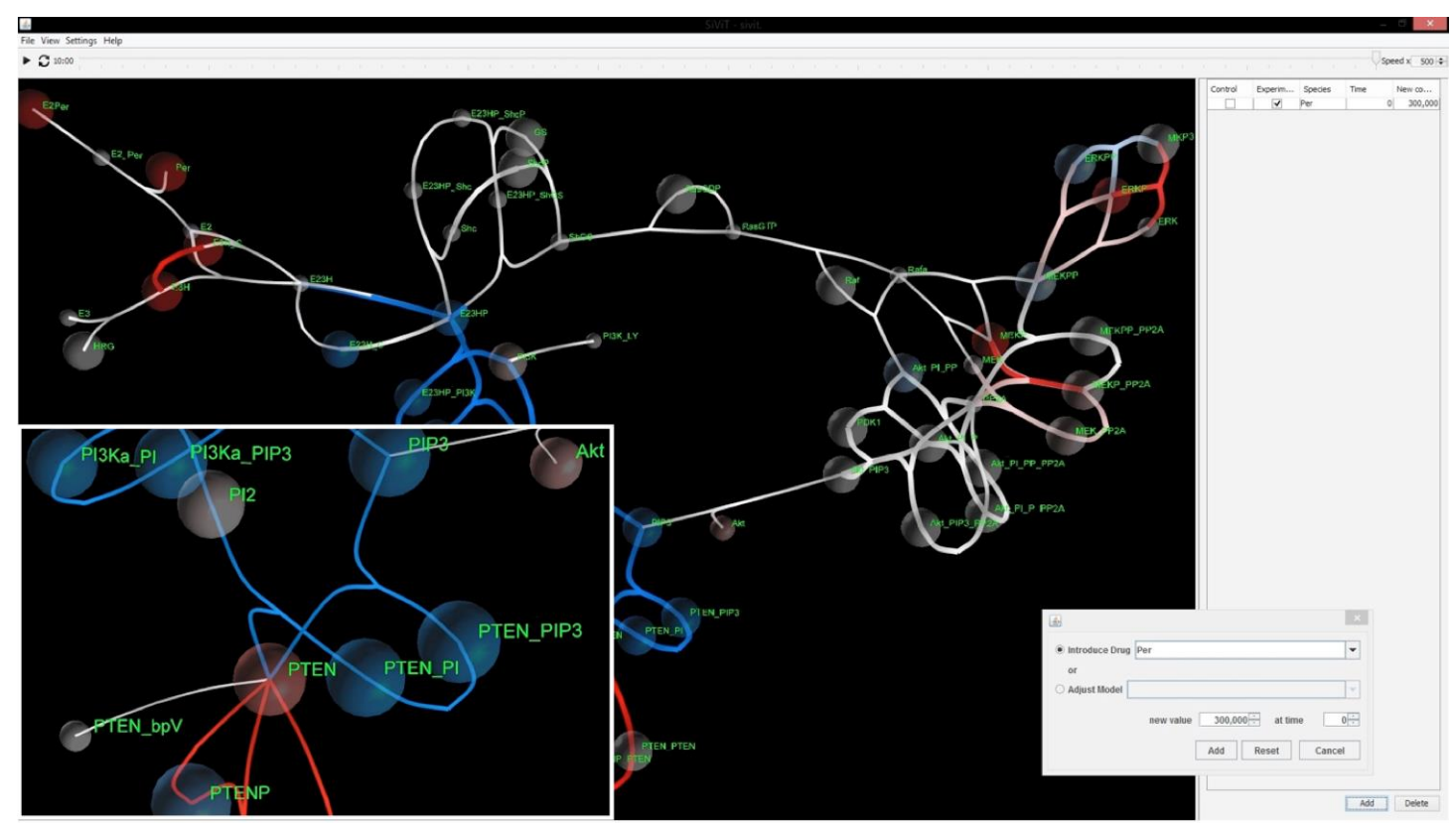

Figure 3: a signaling network visualisation with a pop-up dialogue box (Inset 1, bottom right) for amending drug regime and mutational status together with an inset with magnified detail (Inset 2, bottom left) taken from Bown et al. (2016). See http://WwW.impactjournals.com/oncotarget/index.php?journal=oncotarget\&page= article\&op=view\&path\%5B\%5D=8747 for figures and related movies.

Figure 4 shows a typical set of SiViT visualisations. The network is that of the PI3K/PTEN/AKT and RAF/MEK/ERK pathways shown in Figure 1 (except for the sub-network of Figure 1 below the ERK-pERK-ppERK interactions). Figure 4A and $4 \mathrm{~B}$ show the effect of an anti-cancer drug after 1 minute and 10 minutes respectively. Figure $4 \mathrm{~A}$ shows an immediate and substantial down-regulation of signalling since most of the network is blue. By 10 minutes, we observe differences in pathway dynamics: in Figure 4B the lower pathway is still down-regulated and the upper pathway has similar levels of signalling to the network without the drug, i.e. the Control condition, since much of this pathway is white. Thus, the overall signalling activity in this upper pathway is the same but signalling dynamics are slowed by the drug action - a different dynamic to that of the lower pathway. Areas in red show non-local, emergent phenomena: red areas represent accumulations of species concentrations as a result of drug action inhibiting nodes elsewhere in the pathway network.

Figure 4C shows a network with a cancer-causing mutation introduced at a single point. This single point mutation has a marked effect on whole network functioning, where at the end of the 10-minute time-course the inhibitor has a far weaker effect in reducing signalling. Figure 4D depicts the state of the network after the use of combination therapy to restore network sensitivity to the drug. The resistant network with combination therapy shown in Figure 4D is very similar to 
the sensitive (normal) network with single therapy in Figure 4B in respect of the overall pattern of signalling.

Note, the white insets show key biological indicators of signalling activity in the Control (black line) and Experiment (blue line) simulation conditions. In Figures 4A, 4C and 4D the insets show AKT levels - a key regulator of cell survival, growth and proliferation. The intended drug action is to reduce the amount of active AKT in the network. In Figure 4A levels of inactive AKT are increased following drug action as expected; in the resistant network of Figure $4 \mathrm{C}$ inactive AKT is decreased. In Figure 4D, SiViT was used to determine the minimum dose to match the key biological indicator (AKT) as shown in the overlapping blue and black lines of the pop-up inset.

Beyond this representation, the observer is currently left to construct an interpretation of the system dynamics by integrating the observed individual components; the task is of course easier than such inference from the equation set or graph-based time-series readouts. Additional layers of abstraction and perspectives could add to the explanatory power of simulation results. 

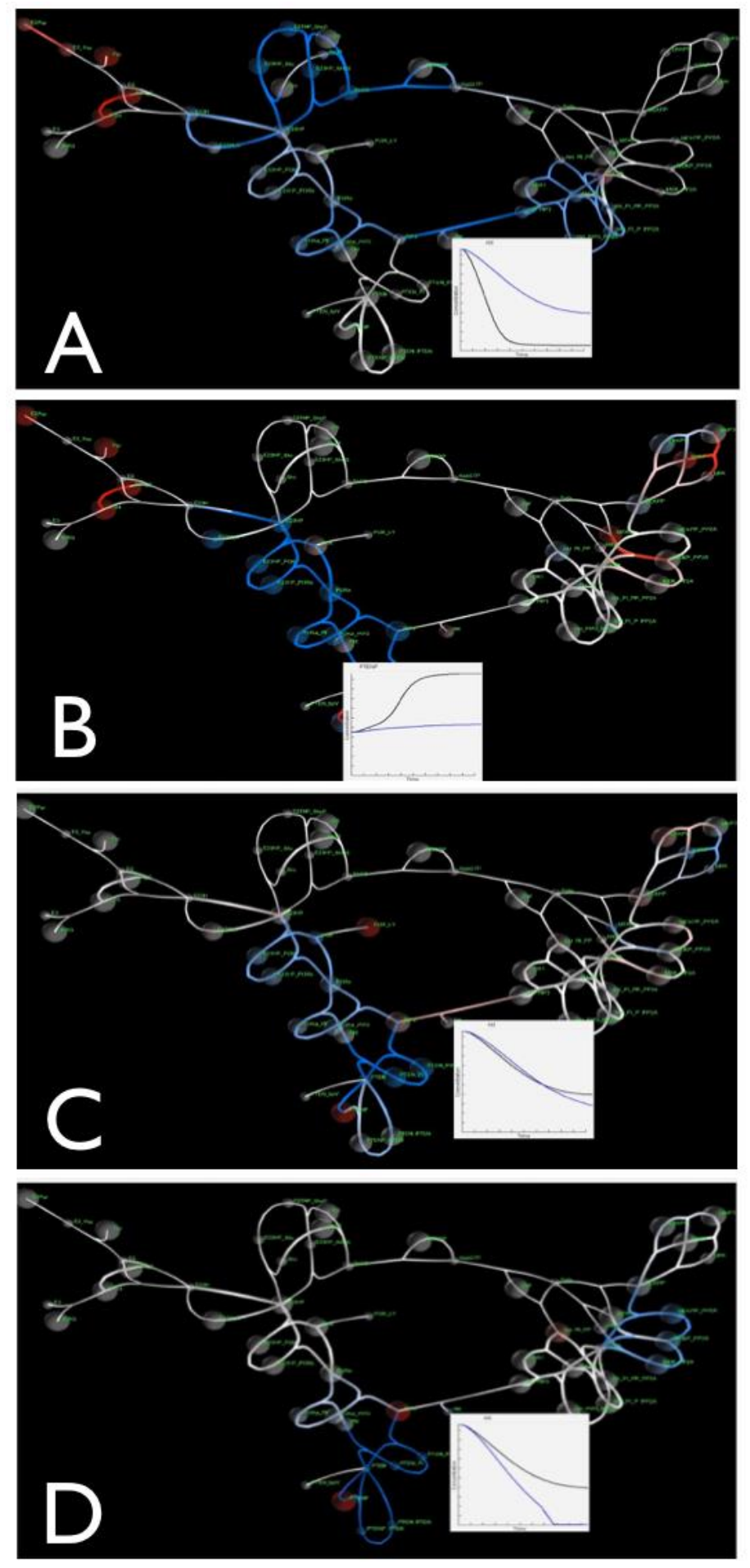

Figure 4: SiViT visualisations of cell signalling (adapted from Bown et al. 2016). Figure $3 A$ and $3 B$ show the effect of an anti-cancer drug after 1 minute and 10 minutes respectively. Figure $3 C$ shows a network with a cancer-causing mutation introduced at a single point. Figure 3D shows the same network as Figure 3C but with combination therapy to overcome resistance. See http://www.impactjournals.com/oncotarget/index.php?journal=oncotarget\&page= article\&op=view\&path\%5B\%5D=8747 for figures and related movies. 


\section{Conclusion}

SiViT can reveal system dynamics in a literal sense. A key limitation of many information visualisation systems identified by (Lee et al. 2015) is that there is no provision for the "making of a story". Where visualisations cue key events in the system dynamics, or story pieces, it is down to the user to extract and organise meaningfully those story pieces without support from the visualisation system (Lee et al. 2015). Additionally, visualisations are not typically designed to highlight key events, including system changes in functioning and - here - the invocation of compensatory mechanisms of feedback.

For signalling networks, in the light of increasing understanding of the limitations of drug design targeting single nodes in the face of topological changes in signalling network architecture, Behar et al. (2013) propose that signalling hub topology, and crucially the response of that topology to differing signalling pathway inputs i.e. drugs, has utility in advancing anti-cancer therapy. In Behar et al. (2013) the network is viewed in terms of sub-networks that provide dynamical mapping of inputs to outputs; interventions (drugs) are viewed in terms of their impact on that mapping. They combine this concept with that of a network motif (Wong et al. 2011), where a motif is a particular configuration of nodes in a subnetwork that is observed regularly and pervasively in a range of biological systems. Motifs include switches, feedback loops, feed-forward loops and integrators. Behar et al. (2013) suggest that signalling networks either exhibit these motifs explicitly or may be abstracted into such a motif based on the observed dynamics of that subnetwork. In taking this view the network, or at least sub-networks, in addition to the pathway nodes, has agency. Moreover, motifs and changes in network or subnetwork topology may well represent key story pieces.

Thus any interactive, visual account of cell signalling in response to drugs needs to reveal both node and sub-network dynamics, in an integrated and concurrent manner. System-scale dynamics must be portrayed such that conformational changes in sub-networks, such as from e.g. a feedback into a feed-forward loop, are depicted concurrently with up- and down-regulation of nodes, and importantly how the two are connected. This interconnection is likely to be nonlinear and context-sensitive: we must explore the use of concurrent accounts of signalling at different spatial and temporal scales to reveal this link, with key events in each account being cross-linked to reveal how one impacts the other.

This dynamic representation of signalling network dynamics requires communication via video, and while highlighting important events is not readily translated into a narrative form. New work by Bach et al. (2016) provides a thought 
provoking first study on the use of the well-established and visually rich medium of comics to tell stories about dynamic networks. Bach et al. (2016) note that comics are already used to convey information beyond entertainment in order to inform and educate in an engaging way. They founded their notational design on good practice in the existing domain of graphic comics and tested the effectiveness of designed comics to convey network changes over time. The results confirmed that with minimal textual cueing the intended dynamics were successfully conveyed.

While the comics used in this study are not as complex as required to represent signalling network dynamics, we believe that this work, combined with our own interactive and executable visualisation technology, provides a first hint towards the narrating of cellular signalling networks. In principle, and with some user direction, key switches in behaviour driven by drug action and mutation identified via SiViT could be pushed out to a comic format with panels showing key states and alternate pathways in time. The comic would then architect the key story pieces, and events leading to marked changes in signalling network functioning, into a narrative underpinned by the more detailed SiViT visualisation. This would both aid understanding of signalling network dynamics and, crucially, improve reporting in linear reporting media and in particular scientific journals. 


\section{References}

Bach B, Kerracher N, Hall K W, Carpendale S, Kennedy J, Riche N. Telling Stories about Dynamic Networks with Graph Comics. In Proceedings of the 2016 CHI Conference on Human Factors in Computing Systems (CHI '16). ACM, New York, NY, USA, 3670-3682. DOI: http://dx.doi.org/10.1145/2858036.2858387.

Behar M, Barken D, Werner SL, Hoffmann A. The Dynamics of Signaling as a Pharmacological Target. Cell. 2013; 155:448-461.

Bown J, Andrews PS, Deeni Y, Goltsov A, Idowu M, Polack FAC, Sampson ATS, Shovman M, Stepney S. Engineering Simulations for Cancer Systems Biology. Current Drug Targets. 2012; 13(12):1560-74.

Bown JL, Shovman M, Robertson P, Boiko A, Goltsov A, Mullen P, Harrison DJ. A signaling visualization toolkit to support rational design of combination therapies and biomarker discovery: SiViT. Oncotarget. 2016; accepted.

Boy J, Detienne F, Fekete JD. Storytelling in Information Visualizations: Does it Engage Users to Explore Data?. InProceedings of the 33rd Annual ACM Conference on Human Factors in Computing Systems 2015 Apr 18 (pp. 1449-1458). ACM.

Cancer Research UK 2016. Accessed at: http://www.cancerresearchuk.org/aboutcancer/what-is-cancer (accessed on 03/8/16).

Chandarlapaty $\mathrm{S}$. Negative feedback and adaptive resistance to the targeted therapy of cancer. Cancer Discov. 2012; 2:311-9.

Chong CR, Jänne PA. The quest to overcome resistance to EGFR-targeted therapies in cancer. Nat Med. 2013; 19:1389-1400.

Cooper GM. The Cell: A Molecular Approach, $2^{\text {nd }}$ Edition. Sunderland (MA): Sinauer Associates (2000).

Coulter A, Entwistle VA, Eccles A, Ryan S, Shepperd S, Perera R. Personalised care planning for adults with chronic or long-term health conditions. Cochrane Database of Systematic Reviews 2015, Issue 3.

Department of Health. Long Term Conditions Compendium of Information: 3rd edition. Available at: https://www.gov.uk/government/publications/longterm-conditions-compendium-of-information-third-edition. 2013 (accessed on 3/8/2016).

Goltsov A, Faratian D, Langdon SP, Mullen P, Harrison DJ, Bown J. Features of the reversible sensitivity-resistance transition in PI3K/PTEN/AKT signaling network after HER2 inhibition. Cell Signal. 2012; 24:493-504.

Goltsov A, Langdon SP, Goltsov G, Harrison DJ, Bown J. Customizing the Therapeutic Response of Signaling Networks to Promote Antitumor Responses by Drug Combinations. Frontiers in Oncology. 2014; 4:13. doi:10.3389/fonc.2014.00013.

Hanahan D, Weinberg RA. Hallmarks of cancer: the next generation. Cell. 2011; 144:646-674. 
Hu H, Gotlsov A, Bown JL, Sims AH, Langdon SP, Harrison DJ, Faratian D.

Feedforward and feedback regulation of the MAPK and PI3K oscillatory

circuit in breast cancer. Cell Signal. 2013; 25(1):26-32.

Jaalouk DE, Lammerding J. Nature Reviews Molecular Cell Biology. 2009; 10:6373. doi:10.1038/nrm2597.

Janes KA, Lauffenburger DA. Models of signalling networks - what cell biologists can gain from them and give to them. J Cell Sci 2013(126):1913-1921.

Janssen $E$, van Osch $L$, de Vries, Lechner, L. The influence of narrative risk communication on feelings of cancer risk. British Journal of Health Psychology. 2013; 18: 407-419. doi: 10.1111/j.2044-8287.2012.02098.x

King's Fund (2012) Time to think differently. Long term conditions and multimorbidity. Available at: http://www.kingsfund.org.uk/time-to-thinkdifferently/trends/disease-and-disability/long-term-conditions-multimorbidity\#morbidity 2012 (accessed on 3/8/2016).

Kholodenko, BN. Drug Resistance Resulting from Kinase Dimerization Is Rationalized by Thermodynamic Factors Describing Allosteric Inhibitor Effects. Cell Rep. 2015; 12:1939-1949. doi: 10.1016/j.celrep.2015.08.014. Kreeger PK, Lauffenburger DA. Cancer systems biology: a net- work modeling perspective. Carciogenesis 2009; 31(1): 2-8.

Lee B, Riche N, Isenberg P, Carpendale S. More than Telling a Story: A Closer Look at the Process of Transforming Data into Visually Shared Stories. IEEE Computer Graphics and Applications 2015; 35(5): 84-90.

Lee MJ, Ye AS, Gardino AK, Heijnk AM, Sorger PK, MacBeath G, Yaffe MB. Sequential application of anticancer drugs enhances cell death by rewiring apoptotic signaling networks. Cell. 2012; 149:780-794.

Medina-Franco JL, Giulianotti MA, Welmaker GS, Houghten RA. Shifting from the single- to the multitarget paradigm in drug discovery. Drug Discov Today. 2013; 18:495-501.

Mortensen LS, Johansen J, Kallehauge JF, Primdahl H, Busk M, Lassen P et al. FAZA $\mathrm{PET} / \mathrm{CT}$ hypoxia imaging in patients with squamous cell carcinoma of the head and neck treated with radiotherapy: Results from the DAHANCA 24 trial. Radiotherapy \& Oncology. 2012 Oct;105(1):14-20.

Segel E, Heer J. Narrative visualization: Telling stories with data. IEEE transactions on visualization and computer graphics. 2010;16(6):1139-48.

Shaffer VA, Hulsey L, Zikmund-Fisher BJ. The effects of process-focused versus experience-focused narratives in a breast cancer treatment decision task. Patient Education and Counseling. 2013; 93(2): 255-264.

Singer CF, Köstler WJ, Hudelist G. Predicting the efficacy of trastuzumab-based therapy in breast cancer: current standards and future strategies. Biochimica et Biophysica Acta.2008; 1786(2):105-13.

Stepney S, Welch PH, Andrews PS, Ritson CG eds. Proceedings of the 2011 Workshop on Complex Systems Modelling and Simulation, Paris, France, August 2011. Luniver Press 2011 
Stewart BW, Wild CP. World Cancer Report WHO Press. 2014.

United Nations General Assembly. Available at:

https://www.idf.org/sites/default/files/UN\%20Secretary\%20General\%20Progr ess\%20Report\%20on\%20NCDs\%20Dec\%202013.pdf 2013 (accessed 3/8/2016).

Wong E, Baur B, Quader S, Huang CH. Biological network motif detection: principles and practice. Brief Bioinform. 2012;13(2):202-15. doi:

10.1093/bib/bbr033. 